\title{
PREVALENCIA Y FACTORES ASOCIADOS A LA COMPRA DE ANTIMICROBIANOS SIN RECETA MÉDICA, PERÚ 2016
}

\author{
Carolay Rojas-Adrianzén ${ }^{1, a}$, Reneé Pereyra-Elías ${ }^{1, a}$, Percy Mayta-Tristán ${ }^{1,2, a}$
}

\begin{abstract}
RESUMEN
Objetivos. Describir la prevalencia y los factores asociados a la compra de antimicrobianos sin receta médica (CASRM) en usuarios de boticas/farmacias según la Encuesta Nacional de Satisfacción de Usuarios en Salud (ENSUSALUD). Materiales y métodos. Se realizó un análisis secundario de la ENSUSALUD 2016; estudio con muestreo probabilístico bietápico en las Instituciones Prestadoras de Servicios de Salud del subsector público y privado. La CASRM se evaluó mediante autorreporte. Se estimaron las razones de prevalencia crudas (RP) y ajustadas (RPa) con intervalos de confianza al 95\% (IC 95\%). Resultados. Se incluyeron 1165 usuarios que compraron antimicrobianos. El promedio de edad fue 38,0 años (desviación estándar: 13,4). La prevalencia de CASRM fue 53,4\% (IC 95\%: 48,0-58,8). La prevalencia de CASRM fue mayor en la sierra y la selva, respecto a Lima Metropolitana (RPa: 1,66; IC 95\%: 1,37-2,02; y RPa: 1,61; IC 95\%: 1,31-1,99; respectivamente); también fue mayor en los usuarios de farmacias respecto a los de boticas (RPa: 1,25; IC 95\%: 1,13-1,39). Las personas con Seguro Integral de Salud (SIS) tuvieron menor prevalencia de CASRM respecto a quienes no tenían seguro (RPa: 0,73; IC 95\%: 0,60-0,88). La prevalencia fue menor si el antimicrobiano era para el cónyuge (RPa: 0,65; IC 95\%: 0,55-0,77) o el hijo(a) (RPa: 0,70; IC 95\%: 0,52-0,93) comparada con si era compraba para uno mismo. Conclusiones. El 53,4\% de usuarios de boticas/farmacias, que compraron antimicrobianos, lo hicieron sin receta médica. La prevalencia de CASRM fue mayor en la sierra y selva y en los usuarios de farmacias, así como menor en quienes contaban con SIS y quienes compraban para su cónyuge o hijo(a). Sugerimos reforzar las intervenciones, principalmente regulatorias y educativas, en la sierra y en la selva, así como, en la población no afiliada a un seguro de salud.
\end{abstract}

Palabras claves: Automedicación; Antibacterianos; Antiinfecciosos; Accesibilidad a los Servicios de Salud; Perú (fuente: DeCS BIREME).

\section{PREVALENCE AND FACTORS ASSOCIATED WITH OVER-THE-COUNTER ANTIMICROBIAL PURCHASES, PERU 2016}

\begin{abstract}
Objectives. To describe the prevalence and factors associated with the purchase of antimicrobials without a prescription (CASRM) in drugstore/pharmacy users according to the National Health User Satisfaction Survey (ENSUSALUD). Materials and Methods. A secondary analysis of ENSUSALUD 2016 was carried out; a study with two-stage probabilistic sampling in the Health Service Providers of the public and private subsector. The CASRM was evaluated by self-report. Crude (PR) and adjusted (PRa) prevalence ratios with 95\% confidence intervals $(95 \% \mathrm{Cl})$ were estimated. Results. The study included 1.165 users who purchased antimicrobials. The mean age was 38.0 years (standard deviation: 13.4). The prevalence of CASRM was 53.4\% (95\% Cl: 48.0-58.8). The prevalence of CASRM was higher in the highlands and jungle, respect to metropolitan Lima (PRa: 1,66; CI 95\%: 1,37-2,02; y PRa: 1,61; Cl 95\%: 1,31-1,99; respectively); also was higher in pharmacy users compared to drugstore users (PRa: 1,25; Cl 95\%: 1,13-1,39). People with comprenhensive health insurance (SIS) had a lower prevalence of CASRM compared to people without insurance (PRa: 0,73; $\mathrm{Cl} 95 \%$ : $0,60-0,88$ ). The prevalence was lower if the antimicrobial was for the spouse (PRa: 0,$65 ; \mathrm{Cl} 95 \%: 0,55-0,77$ ) or child (PRa: 0,70; IC 95\%: 0,52-0,93) compared to if it was buying for oneself. Conclusions. A $53.4 \%$ of drugstore/pharmacy users, who bought antimicrobials, did so without a doctor's prescription. The prevalence of CASRM was higher in the highlands and jungle and in pharmacy users, as well as lower in those who had SIS and those who bought for their spouse or child. We suggest reinforcing the interventions, mainly regulatory and educational, in the highlands and in the jungle, as well as, in the population not affiliated to a health insurance.
\end{abstract}

Keywords: Self-medication; Antibacterial; anti-infective; Accessibility to health services; Perú (source: MeSH NLM).

Escuela de Medicina, Universidad Peruana de Ciencias Aplicadas, Lima, Perú.

Dirección General de Investigación, Desarrollo e Innovación, Universidad Científica del Sur, Lima, Perú.

Médico cirujano.

Recibido: 06/02/2018 Aprobado: 20/06/2018 En línea: 11/09/2018

Citar como: Rojas-Adrianzén C, Pereyra-Elías R, Percy Mayta-Tristán P. Prevalencia y factores asociados a la compra de antimicrobianos sin receta médica, Perú 2016. Rev Peru Med Exp Salud Publica. 2018;35(3):400-8. doi: 10.17843/rpmesp.2018.353.3458. 


\section{INTRODUCCIÓN}

La automedicación con antibióticos es un problema de salud pública a nivel global ${ }^{(1-3)}$. La automedicación se define como aquella práctica en la que se adquiere y consume algún medicamento sin la opinión o supervisión médica ${ }^{(4)}$. Así, incluye volver a usar recetas médicas para adquirir medicamentos, compartir medicamentos entre los miembros de una familia o amigos o usar medicamentos que quedaron de una compra antigua ${ }^{(4)}$. Los medicamentos que con mayor frecuencia se compran sin receta médica son los analgésicos seguido de los fármacos para síntomas de resfrío común, para síntomas gastrointestinales y los antibióticos ${ }^{(5-8)}$. El uso irracional y descontrolado de antibióticos puede tener consecuencias negativas como la falta de respuesta al tratamiento, efectos adversos y la resistencia antibiótica ${ }^{(1,2,9,10)}$. Dentro de esta última, la selección de bacterias multidrogorresistentes puede resultar en una duración prolongada de la enfermedad, más visitas al médico, más días de hospitalización, necesidad de medicamentos caros, tóxicos y la muerte ${ }^{(2,3,11,12)}$.

Existen diversos factores asociados a la compra de antibióticos sin receta médica (CASRM), así como los antibióticos que se consumen con más frecuencia y las razones por las que se opta por la automedicación con estos ${ }^{(2,5,13)}$. Entre estos factores, se encuentran ser de mediana edad (40-59 años) ${ }^{(13)}$, el bajo nivel educativo ${ }^{(2,13)}$, el sexo masculino ${ }^{(5,8,12,13)}$, el menor nivel de ingresos ${ }^{(13)}$, entre otros. Dentro de los antibióticos, que más se consumen están los betalactámicos ${ }^{(5,8,12,13)}$; seguido por los macrólidos, las quinolonas y las tetraciclinas ${ }^{(13)}$. Por otra parte, las principales razones para recurrir a la automedicación con antibióticos serían la falta de recursos para acceder a servicios de salud, la falta de regulación en la adquisición de estos fármacos, así como el desconocimiento del fenómeno de resistencia antimicrobiana y otras potenciales consecuencias del uso de estas drogas ${ }^{(8)}$.

Respecto a la regulación en la adquisición de antibióticos, es fundamental que los países tengan políticas claras para su buen uso ${ }^{(14)}$. En el Perú, la Ley General de Salud $N^{\circ} 26842{ }^{\left({ }^{15}\right)}$ específica que la venta de antibióticos sólo se da bajo la presentación de receta médica y el Ministerio de Salud está obligado a constatar que esto se cumpla. A pesar de esto, la prevalencia de venta de antibióticos sin receta médica es muy alta, la cual oscila entre un $25,0 \%$ a $58,0 \%(14,16,17)$. La alta prevalencia de este comportamiento podría explicarse, porque la población peruana aún presenta ciertas barreras de acceso a los servicios de salud ${ }^{(18)}$, entre otros factores.

Sin embargo, a pesar de la importancia del tema, en el Perú, existen estudios escasos, de pequeños tamaños muestrales o con sesgos metodológicos importantes. En ese sentido, se considera relevante desarrollar el presente

\section{MENSAJES CLAVE}

Motivación para realizar el estudio. La resistencia a los antibióticos es un problema de salud pública a nivel global, y una de las causas es el uso indiscriminado de estos a través de la automedicación.

Principales hallazgos. El 53,4\% de usuarios que compraron antimicrobianos en boticas/farmacias del Perú, lo hicieron sin receta médica. La prevalencia fue mayor en la sierra y selva, y menor en quienes contaban con seguro y quienes compraban para su cónyuge o hijo(a).

Implicancias. Se debe asegurar un control estricto del expendio de antibióticos (en especial, en la sierra y en la selva), además de implementar medidas educativas para la población.

análisis que tiene como objetivo describir la prevalencia y los factores asociados a la compra de antimicrobianos sin receta médica (CASRM) en usuarios de boticas/ farmacias según la Encuesta Nacional de Satisfacción de Usuarios en Salud (ENSUSALUD), Perú 2016.

\section{MATERIALES Y MÉTODOS}

\section{DISEÑO Y ESTUDIO PRIMARIO}

Se realizó un análisis secundario de datos del «Cuestionario 4» (dirigido a usuarios de farmacias y boticas) (C4FYB) de la ENSUSALUD 2016, la cual fue desarrollada en convenio con la Superintendencia Nacional de Salud (SUSALUD) y el Instituto Nacional de Estadística e Informática (INEI) desde el 13 de mayo hasta el 9 de julio del $2016{ }^{(19)}$. El estudio es de tipo observacional, transversal y analítico.

\section{POBLACIÓN, MUESTRA Y MUESTREO}

La encuesta incluyó a usuarios de farmacias y boticas mayores de 15 años que compraron algún medicamento para ellos mismos, su cónyuge o hijos en las farmacias o boticas que se encontraban en un perímetro de hasta dos cuadras de las Instituciones Prestadoras de Servicios de Salud (IPRESS) distribuidas entre el subsector público conformados por los hospitales del Ministerio de Salud y Gobierno Regional (MINSA-GR), Seguro Social en Salud (EsSalud) y las Sanidades de las Fuerzas Armadas y Policiales (FF.AA y PNP); y las clínicas del subsector privado (CSP) cuya selección fue probabilística, estratificada y bietápica. La unidad primaria de muestreo fueron las IPRESS, las cuales fueron seleccionadas de manera sistemática con probabilidad proporcional al número de atendidos, la unidad secundaria de muestreo fueron los usuarios de farmacias y boticas, cuya selección fue aleatoria. Se tuvieron 25 estratos que correspondieron a los 24 departamentos del Perú y a la provincia constitucional del Callao. En base a ello, se estimaron los factores de expansión ${ }^{(19)}$. 


\section{VARIABLES Y MEDICIONES}

Mediante la pregunta 11 del C4BYF: ¿Qué medicamento/s compró usted?, se creó la variable antimicrobianos en base a la lista de medicamentos (genéricos y comerciales). Seleccionamos a los participantes que hubieran adquirido por lo menos un antimicrobiano en su compra. La variable respuesta se evaluó mediante la pregunta «"Este/estos medicamentos, ¿Usted lo/s compró con receta?" y las alternativas de respuestas fueron: 1) Sí y muestra receta 2) Sí y no muestra receta y, 3) No». Para el análisis, se consideró a las alternativas 1 y 2 como compra de antimicrobianos con receta médica.

Las variables estudiadas como predictoras fueron características sociodemográficas del usuario, que consistían en edad (jóvenes de 15 a 29 años, adulto de 30 a 59 años y adulto mayorde 60 años a más), sexo (hombre/mujer), región natural (Lima Metropolitana, el resto de la Costa, Sierra, Selva), grado de instrucción (primaria completa o menor, secundaria completa, técnica completa y superior universitaria completa o posgrado), idioma (español o quechua y lenguas aborígenes), actividad laboral (no trabaja, trabaja, estudia), ingreso familiar mensual (de 100 a 900 soles, de 950 a 1500 soles y de 1600 a 10000 soles), tipo de seguro de salud (el cual se categorizó en: sin ningún seguro; Seguro Integral de Salud (SIS); EsSalud; FF.AAy PNP; CSP, donde se incluyeron a los seguros de las entidades prestadoras de salud, seguro de salud de aseguradoras privadas, seguro de salud de las clínicas y seguro universitario; y una categoría adicional para usuarios con más de un seguro). También, se evaluó si el antimicrobiano se adquirió en una farmacia o botica (las farmacias fueron definidas como los establecimientos de propiedad de un profesional químico farmacéutico). Además, se incluyó una variable que evaluaba para quién eran el/los medicamentos que estaba comprando el usuario (para sí mismo, para su cónyuge o para su hijo/hija).

\section{ANÁLISIS ESTADÍSTICO}

La base de datos fue importada a Stata ${ }^{\circledR}$ versión 12.0 (Stata Corporation, College Station, Texas, USA) y fue adecuada al tipo de muestreo de ENSUSALUD 2016, incluyendo las ponderaciones de acuerdo a estratos, conglomerados, factores de expansión y efecto de diseño. Se utilizó el módulo de análisis de muestras complejas (Survey complex data-analysis: svy).

En el análisis descriptivo, se calcularon las frecuencias absolutas y relativas de las variables categóricas. Se categorizaron las variables numéricas edad e ingreso familiar mensual. En el análisis bivariado, se evaluó la asociación entre las variables categóricas mediante la prueba de chi-cuadrado, previa evaluación de los valores esperados. Se realizaron modelos lineales generalizados (GLM) de familia Poisson tomando como variable de respuesta a la CASRM. Además, se realizó un modelo ajustado, que incluyó aquellas variables con $p<0,20$ en el likelihood ratio test del modelo crudo comparado con el modelo nulo. Se reportan las razones de prevalencia crudas (RP) y ajustadas (RPa) con intervalos de confianza al 95\% (IC 95\%). Todos los análisis fueron realizados con un nivel de significancia del $5 \%$.

\section{ASPECTOS ÉTICOS}

El protocolo de investigación del presente estudio fue enviado al Comité de Ética e Investigación de la Facultad de Ciencias de Salud de la Universidad Peruana de Ciencias Aplicadas. Al ser un análisis secundario de una base de datos pública y sin identificadores, el estudio fue exonerado de revisión.

\section{RESULTADOS}

\section{CARACTERÍSTICAS DE LA POBLACIÓN}

El C4FYB registró un total de 3858 cuestionarios completos, de los cuales 1165 (30,2\%) fueron incluidos en el análisis (Figura 1). Estos 1165 usuarios de boticas y farmacias que compraron antimicrobianos representan a una población expandida de 863469 usuarios a nivel nacional. La media de la edad fue 38,0 años (d.e.: 13,4 ) y el $62,0 \%$ de la población eran adultos entre 30 a 59 años de edad. Aproximadamente, el $57,5 \%$ fueron mujeres. El $16,2 \%$ usuarios de farmacias y, el resto de boticas. El $35,8 \%$ de los usuarios vivían en la selva, el 25,3\% de Lima Metropolitana,

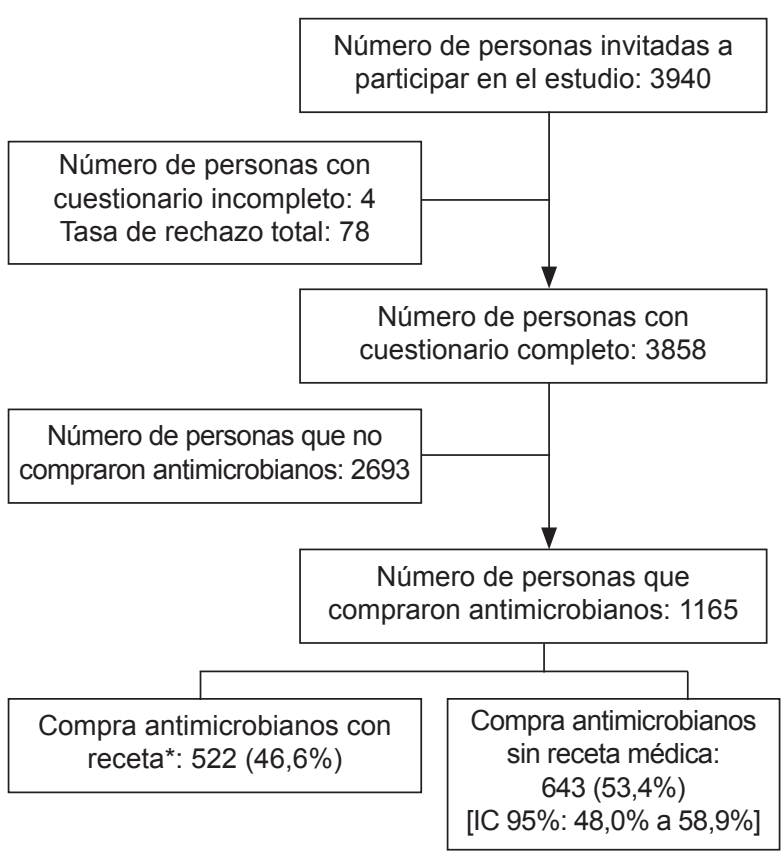

Figura 1. Flujograma de participación en el estudio: Prevalencia y factores asociados a la compra de antimicrobianos sin receta médica en usuarios de farmacias y boticas en Perú, 2016.

* Se consideró al grupo de usuarios que compraron medicamentos con receta y la mostraron, así como a aquellos usuarios que dijeron tener receta, pero no la mostraron. 
$22,6 \%$ de la costa y el $16,3 \%$ de la sierra. El $66,8 \%$ de los encuestados tenían secundaria completa como mínimo. La mayor proporción de asegurados se encontraban afiliados al SIS $(34,7 \%)$, seguido por EsSalud $(24,8 \%)$, mientras que el $35,2 \%$ de la población refirió no tener seguro de salud. Por otra parte, el $65,6 \%$ de los usuarios que CASRM lo realizó para si mísmo. Finalmente, la prevalencia de la CASRM en la población de estudio fue del 53,4\% [IC95\%: 48,0\%-58,9\%]. (Tabla 1).

\section{ANÁLISIS BIVARIADO}

En el análisis bivariado, respecto a la región natural de procedencia, los participantes de la sierra y selva tuvieron mayor prevalencia de haber CASRM $(66,1 \%$ y $63,5 \%$ respectivamente) en comparación con quienes lo compraron en Lima Metropolitana (42,3\%; $p<0,001)$. Respecto al tipo de seguro, la prevalencia de CASRM en los participantes que contaban con SIS fue $43,8 \%$, mientras que esta se encontraba entre $30-55 \%$ en quienes contaban con otros seguros y $62,3 \%$ en quienes no tenían ningún tipo de seguro.

La proporción de CASRM fue mayor en los participantes que lo adquirieron en farmacias en comparación con quienes los adquirieron en boticas $(61,2 \%$ vs $51,9 \%$; $p=0,020$ ). Finalmente, los participantes que compraron el antimicrobiano para sí mismos tuvieron mayor probabilidad de haberlo realizado sin receta médica $(60,6 \%)$ en comparación con quienes lo compraron para sus hijos o cónyuges $(39,0 \%$ y $41,1 \%$ respectivamente; $p<0,001)$. No se encontró diferencia estadísticamente significativa entre la proporción de CASRM según el sexo, grado de instrucción, idioma, ingreso mensual familiar. (Tabla 2)

En el modelo crudo, las variables que mostraron asociación con la CASRM fueron la edad, la región natural, el tipo de seguro de salud y para quién compra el antimicrobiano. Así, la prevalencia de CASRM en usuarios con rango de edad entre 30 a 59 años fue $15,0 \%$ menor a la prevalencia de CASRM en usuarios con rango de edad entre 15 a 29 años. Las prevalencias de CASRM en usuarios de la Sierra/ Selva fueron $56,0 \%$ y $50,0 \%$ mayor, respectivamente, que la prevalencia de la CASRM en los usuarios de Lima Metropolitana. Además, la prevalencia de CASRM en los usuarios con SIS fue $30,0 \%$ menor que en aquellos usuarios sin seguro de salud. Finalmente, los usuarios que compraron medicamentos para su cónyuge o para su hijo/a tuvieron una prevalencia $36,0 \%$ y $32,0 \%$ menor, en comparación de aquellos usuarios que comprar antimicrobianos para ellos mismos.

\section{ANÁLISIS DE MÚLTIPLES VARIABLES}

Para el modelo ajustado, se incluyeron a las variables del modelo crudo con Likelihood ratio test menor a 0,20,
Tabla 1. Características de los usuarios de farmacias/ boticas que compran antimicrobianos. $(n=1165)$

\begin{tabular}{|c|c|c|}
\hline Variables & $\mathrm{n}$ & $\%$ \\
\hline \multicolumn{3}{|l|}{ Edad (años) } \\
\hline $15-29$ & 344 & 29,3 \\
\hline $30-59$ & 732 & 62,0 \\
\hline$\geq 60$ & 89 & 8,7 \\
\hline \multicolumn{3}{|l|}{ Sexo } \\
\hline Mujer & 668 & 57,5 \\
\hline Hombre & 497 & 42,5 \\
\hline \multicolumn{3}{|l|}{ Región natural } \\
\hline Lima metropolitana & 130 & 25,3 \\
\hline Costa & 347 & 22,6 \\
\hline Sierra & 237 & 16,3 \\
\hline Selva & 451 & 35,8 \\
\hline \multicolumn{3}{|l|}{ Grado de Instrucción } \\
\hline Primaria completa o menor & 196 & 20,2 \\
\hline Secundaria completa & 467 & 46,6 \\
\hline Técnica completa & 140 & 14,3 \\
\hline Superior completa o posgrado & 189 & 18,8 \\
\hline \multicolumn{3}{|l|}{ Idioma } \\
\hline Español & 1137 & 98,3 \\
\hline Quechua y lenguas aborígenes & 28 & 1,7 \\
\hline \multicolumn{3}{|l|}{ Actividad laboral } \\
\hline No trabaja & 40 & 3,1 \\
\hline Trabaja & 1038 & 89,5 \\
\hline Estudia & 87 & 7,4 \\
\hline \multicolumn{3}{|l|}{ Ingreso familiar mensual* (soles) } \\
\hline $100-900$ & 357 & 33,3 \\
\hline$>900$ a 1500 & 403 & 36,5 \\
\hline$>1500$ a 10000 & 302 & 30,2 \\
\hline \multicolumn{3}{|l|}{ Tipo de seguro de salud** } \\
\hline No tiene seguro & 378 & 35,2 \\
\hline Con más de un seguro & 15 & 1,6 \\
\hline SIS & 434 & 34,7 \\
\hline Essalud & 300 & 24,8 \\
\hline CSP & 17 & 2,1 \\
\hline FF.AA. y PNP & 14 & 1,6 \\
\hline \multicolumn{3}{|l|}{ Tipo de establecimiento } \\
\hline Botica & 970 & 83,7 \\
\hline Farmacia ${ }^{*}$ & 195 & 16,3 \\
\hline \multicolumn{3}{|l|}{ Para quién compra } \\
\hline Para sí mismo & 763 & 65,6 \\
\hline Para su hijo(a) & 275 & 22,9 \\
\hline Para su cónyuge & 127 & 11,5 \\
\hline
\end{tabular}

*En terciles

** Seguro Integral de Salud (SIS) al cual se accede en establecimientos del Ministerio de Salud y Gobiernos Regionales (MINSA-GR), Seguro Social de Salud (EsSalud), Fuerzas Armadas y Policiales (FF.AA y PNP), y entidades prestadoras de salud, aseguradoras privadas, clínicas privadas y seguro universitario (CSP).

* Se denomina farmacia al establecimiento que es de propiedad de un profesional Químico Farmacéutico.

Todas las estadísticas presentadas consideran la naturaleza compleja del muestreo. 
Tabla 2. Prevalencia y factores asociados a la compra de antimicrobianos sin receta médica en usuarios de farmacias/ boticas según ENSUSALUD 2016

\begin{tabular}{|c|c|c|c|}
\hline Variables & $\begin{array}{c}\text { Sin receta } \\
\mathrm{n}(\%)\end{array}$ & $\begin{array}{c}\text { Con receta } \\
\text { n (\%) }\end{array}$ & Valor de $\mathrm{p}^{*}$ \\
\hline \multicolumn{4}{|l|}{ Edad (años) } \\
\hline $15-29$ & $212(59,8)$ & $132(40,2)$ & 0,028 \\
\hline $30-59$ & $386(50,7)$ & $346(49,3)$ & \\
\hline$\geq 60$ & $45(52,0)$ & $44(48,4)$ & \\
\hline \multicolumn{4}{|l|}{ Sexo } \\
\hline Mujer & $342(50,8)$ & $326(49,2)$ & 0,088 \\
\hline Hombre & $301(57,0)$ & $196(43,0)$ & \\
\hline \multicolumn{4}{|l|}{ Región natural } \\
\hline Lima metropolitana & $55(42,3)$ & $75(57,7)$ & $<0,001$ \\
\hline Costa & $155(40,9)$ & $192(59,1)$ & \\
\hline Sierra & $159(66,1)$ & $78(33,9)$ & \\
\hline Selva & $274(63,5)$ & $177(36,5)$ & \\
\hline \multicolumn{4}{|l|}{ Grado de instrucción } \\
\hline Primaria completa o menor & $134(53,8)$ & $120(46,2)$ & 0,354 \\
\hline Secundaria completa & $300(51,8)$ & $239(48,2)$ & \\
\hline Técnica completa & $79(47,6)$ & $89(52,4)$ & \\
\hline Superior completa o posgrado & $130(61,5)$ & $74(38,5)$ & \\
\hline \multicolumn{4}{|l|}{ Idioma } \\
\hline Español & $630(53,5)$ & $507(46,5)$ & 0,790 \\
\hline Quechua y lenguas aborígenes & $13(50,9)$ & $15(49,1)$ & \\
\hline \multicolumn{4}{|l|}{ Actividad laboral } \\
\hline No trabaja & $21(55,8)$ & $19(44,2)$ & 0,035 \\
\hline Trabaja & $563(52,3)$ & $475(47,7)$ & \\
\hline Estudia & $59(66,5)$ & $28(33,5)$ & \\
\hline \multicolumn{4}{|l|}{ Ingreso familiar mensual (soles) } \\
\hline $100-900$ & $179(50,4)$ & $178(49,6)$ & 0,785 \\
\hline$>900$ a 1500 & $230(54,2)$ & $173(45,8)$ & \\
\hline$>1500$ a 10000 & $167(51,3)$ & $135(48,7)$ & \\
\hline \multicolumn{4}{|l|}{ Tipo de seguro de salud** } \\
\hline No tiene seguro & $236(62,3)$ & $142(37,7)$ & 0,004 \\
\hline Con más de un seguro & $9(55,1)$ & $6(44,9)$ & \\
\hline SIS & $202(43,8)$ & $232(56,2)$ & \\
\hline EsSalud & $178(56,0)$ & $122(44,0)$ & \\
\hline CSP & $7(39,5)$ & $10(60,5)$ & \\
\hline FF.AA. y PNP & $4(30,3)$ & $10(69,7)$ & \\
\hline \multicolumn{4}{|l|}{ Tipo de establecimiento } \\
\hline Botica & $516(51,9)$ & $454(48,1)$ & 0,020 \\
\hline Farmacia ${ }^{*}$ & $127(61,2)$ & $68(38,8)$ & \\
\hline \multicolumn{4}{|l|}{ Para quién compra: } \\
\hline Para sí mismo & $470(60,6)$ & $293(39,4)$ & $<0,001$ \\
\hline Para su hijo(a) & $115(39,0)$ & $160(61,0)$ & \\
\hline Para su cónyuge & $58(41,1)$ & $69(58,9)$ & \\
\hline
\end{tabular}

*Hace referencia a la significancia estadística obtenida a partir de la comparación de proporciones mediante la prueba chi-cuadrado entre las categorías de la variable. Todos los análisis consideran la naturaleza compleja de la muestra.

** Seguro Integral de Salud (SIS) al cual se accede en establecimientos del Ministerio de Salud y Gobiernos Regionales (MINSA-GR), Seguro Social de Salud (EsSalud), Fuerzas Armadas y Policiales (FF.AA. y PNP), y entidades prestadoras de salud privadas, clínicas privadas y seguro universitario (CSP).

* Se denomina farmacia al establecimiento que es de propiedad de un profesional químico farmacéutico.

Los $\mathrm{n}$ demuestran los valores absolutos en la muestra; las proporciones fueron ponderadas por el diseño complejo de la muestra. 
incluyendo la edad y el sexo. Las prevalencias de CASRM en usuarios de la Sierra/Selva fueron 66,0\% (RPa: 1,66; IC95\%: 1,37-2,02) y 61,0\% (RPa: 1,61; IC95\%: 1,31-1,99) mayor, respectivamente, que la prevalencia en los usuarios de Lima Metropolitana. Además, la prevalencia de CASRM en los usuarios con SIS fue 27,0\% menor que en aquellos usuarios que no tenían seguro de salud (RPa: 0,73; IC95\%: 0,60-0,88). Por otro lado, los usuarios de farmacias tuvieron una mayor prevalencia de CASRM en comparación a los usuarios de boticas (RPa: 1,25; IC95\%:1,13-1,39). Finalmente, los usuarios que CASRM para su cónyuge o para su hijo/a tuvieron una prevalencia 35,0\% [RPa: 0,65; IC95\%: 0,55-0,77) y 30,0\% (RPa: 0,70; IC95\%: 0,52-0,93) menor, respectivamente, que en aquellos usuarios que compararon el medicamento para ellos mismos (Tabla 3).

\section{DISCUSIÓN}

Los resultados muestran que más de la mitad de usuarios de boticas y farmacias compraron antimicrobianos sin receta médica. Asimismo, los factores que se asociaron a una mayor prevalencia de CASRM fueron ser de la sierra o de la selva, no tener seguro de salud, haber adquirido el antimicrobiano en una farmacia y que el antimicrobiano comprado fuera para ellos mismos.

En nuestro estudio, la prevalencia de CASRM fue de $53,4 \%$. Esta es bastante mayor a la reportada por diversos estudios a nivel mundial ${ }^{(13,20)}$, así como mayor a lo reportado para el Perú por el Comité de Expertos encargados del monitoreo y evaluación del Plan Global contra la resistencia bacteriana $(25 \%)^{(14)}$. El Comité de Expertos encargados del monitoreo y evaluación del Plan Global en contra de la resistencia bacteriana, el cual reportó una prevalencia de automedicación del $25,0 \%{ }^{(14)}$. La diferencia de frecuencias encontrada con otros estudios previos puede deberse a la forma de medición y a las estrategias de selección de muestreo usadas, ya que la mayoría de estas investigaciones utilizó muestreos no probabilísticos ${ }^{(1,2)}$.

La prevalencia de CASRM en los participantes de la sierra y de la selva fue mayor a la prevalencia de CASRM en los usuarios de Lima Metropolitana. Asimismo, la literatura ha reportado prevalencias altas de automedicación en estas regiones del Perú, 85,2\% para un distrito de Cajamarca, un departamento de la sierra ${ }^{(21)}$ y $95,1 \%$ para un distrito de Loreto, un departamento de la selva peruana ${ }^{222}$. Por otro lado, se tiene que la prevalencia de automedicación en un distrito de Lima Metropolitana es de 56,7\% similar a la prevalencia reportada en el presente estudio $(54,4 \%)^{(16)}$.

Es probable a que esta práctica sea más prevalente en la sierra y en la selva por una regulación menos estricta en comparación a Lima y el resto de la costa. Sin embargo, esta es una hipótesis que debe ser confirmada con estudios posteriores. En la literatura, se reportan factores asociados a automedicación similares tanto para los habitantes de la sierra, selva como para los de la costa, y dentro de ella, los de Lima Metropolitana. Estos factores son el tener un rango de edad entre 18 a 35 años, ser de sexo masculino, tener como ocupación la de ser estudiante o empleado y haber recibido consejo de automedicación de algún farmacéutico/boticario o ellos mismos haber decidido el antimicrobiano a tomar $(17,21,22)$.

La prevalencia de CASRM en los usuarios con SIS fue $27,0 \%$ menor que en aquellos usuarios que CASRM y no tenían seguro de salud. La automedicación puede estar favorecida por las características del sistema de salud en el Perú, como es la inaccesibilidad de un alto porcentaje de la población a la asistencia sanitaria como sucede en otros países de Latinoamérica ${ }^{(23)}$. En un estudio en seis países de Latinoamérica, los autores atribuyen la alta prevalencia de automedicación al pobre acceso a servicios de salud ${ }^{(24)}$. Asimismo, en una revisión sistemática realizada por Alhomoud et al (13), se concluyó que la falta de seguro de salud o la falta de acceso a servicios de salud empujarían a algunos individuos a automedicarse como una medida de ahorro al evitar pagar la consulta médica, obteniendo medicamentos de familiares o utilizando aquellos que sobraron de una compra previa (13). Por el contrario, un estudio realizado por Fajardo-Zapata et al, en el 2013, reportó que las personas que se encontraban afiliadas a la seguridad social se automedicaban en un $68,7 \%$ más, en comparación con las que no estaban aseguradas $(23,9 \%)^{(7)}$.

La prevalencia de CASRM en usuarios de farmacias fue mayor en comparación a la prevalencia en usuarios de boticas. Este hallazgo resultó interesante para los autores a la luz de la hipótesis de que la prevalencia de CASRM era mayor en contextos de menor regulación. Sin embargo, podría deberse a que la adquisición de antimicrobianos por recomendación del farmacéutico no es una práctica poco frecuente en nuestro contexto ${ }^{(25)}$. Es necesaria mayor investigación en este punto, para poder dirigir correctamente los esfuerzos en materia regulatoria.

Finalmente, los usuarios que CASRM para su cónyuge o para su hijo/a tuvieron una prevalencia de $34,0 \%$ y $35,0 \%$ menor, respectivamente, que aquellos que CASRM para ellos mismos. La menor prevalencia de CASRM en aquellos que compraron para sus hijos, podría deberse a diversos factores, dentro de ellos, a padres más informados acerca del uso racional de antibióticos. En un estudio realizado por Palmer y Bauchner (26), en el que describieron las opiniones y preocupaciones de los padres por el uso de antibióticos, se encontró que el $29,0 \%$ de los padres estaban preocupados 
Tabla 3. Prevalencia y factores asociados a la compra de antimicrobianos sin receta médica en usuarios de farmacias/ boticas según ENSUSALUD 2016

\begin{tabular}{|c|c|c|}
\hline & Modelo crudo & Modelo ajustado* \\
\hline & RP (IC 95\%) & $\mathrm{RPa}(\mathrm{IC} 95 \%)$ \\
\hline \multicolumn{3}{|l|}{ Edad (años) } \\
\hline $15-29$ & ref. & ref. \\
\hline $30-59$ & $0,85(0,75-0,96)$ & $0,87(0,79-0,97)$ \\
\hline$\geq 60$ & $0,87(0,71-1,07)$ & $0,94(0,76-1,16)$ \\
\hline \multicolumn{3}{|l|}{ Sexo } \\
\hline Mujer & ref. & ref. \\
\hline Hombre & $1,12(0,98-1,28)$ & $1,04(0,91-1,19)$ \\
\hline \multicolumn{3}{|l|}{ Región natural } \\
\hline Lima metropolitana & ref. & ref. \\
\hline Costa & $0,97(0,74-1,27)$ & $1,01(0,81-1,24)$ \\
\hline Sierra & $1,56(1,21-2,01)$ & $1,66(1,37-2,02)$ \\
\hline Selva & $1,50(1,17-1,93)$ & $1,61(1,31-1,99)$ \\
\hline \multicolumn{3}{|l|}{ Grado de instrucción } \\
\hline Primaria completa o menor & ref. & No incluidō \\
\hline Secundaria completa & $0,96(0,82-1,13)$ & \\
\hline Técnica completa & $0,89(0,64-1,22)$ & \\
\hline Superior completa o postgrado & $1,14(0,88-1,49)$ & \\
\hline \multicolumn{3}{|l|}{ Idioma } \\
\hline Español & ref. & No incluidō \\
\hline Quechua y lenguas aborígenes & $1,05(0,72-1,54)$ & \\
\hline \multicolumn{3}{|l|}{ Actividad laboral } \\
\hline No trabaja & ref. & No incluidō \\
\hline Trabaja & $0,94(0,69-1,28)$ & \\
\hline Estudia & $1,19(0,85-1,66)$ & \\
\hline \multicolumn{3}{|l|}{ Ingreso mensual familiar (soles) } \\
\hline $100-900$ & ref. & No incluidō̄ \\
\hline$>900$ a 1500 & $1,07(0,88-1,32)$ & \\
\hline$>1500$ a 10000 & $1,02(0,75-1,38)$ & \\
\hline \multicolumn{3}{|l|}{ Tipo de seguro de salud** } \\
\hline No tiene seguro & ref. & ref. \\
\hline Con más de un seguro & $0,88(0,56-1,40)$ & $0,92(0,62-1,37)$ \\
\hline SIS & $0,70(0,58-0,85)$ & $0,73(0,60-0,88)$ \\
\hline Essalud & $0,90(0,73-1,11)$ & $0,92(0,78-1,08)$ \\
\hline CSP & $0,63(0,37-1,07)$ & $0,76(0,41-1,42)$ \\
\hline FF.AA. y PNP & $0,49(0,18-1,34)$ & $0,54(0,16-1,76)$ \\
\hline \multicolumn{3}{|l|}{ Tipo de establecimiento } \\
\hline Botica & ref. & ref. \\
\hline Farmacia ${ }^{*}$ & $1,18(1,03-1,35)$ & $1,25(1,13-1,39)$ \\
\hline \multicolumn{3}{|l|}{ Para quién compra } \\
\hline Sí mismo & ref. & ref. \\
\hline Su cónyuge & $0,64(0,55-0,75)$ & $0,65(0,55-0,77)$ \\
\hline Su hijo(a) & $0,68(0,49-0,94)$ & $0,70(0,52-0,93)$ \\
\hline
\end{tabular}

\footnotetext{
*Se realizó un modelo lineal generalizado de familia Poisson para muestras complejas.⿳一巛 Sólo se incluyeron a las variables con likelihood ratio test menor a 0,20 en el análisis crudo.

** Seguro Integral de Salud (SIS) al cual se accede en establecimientos del Ministerio de Salud y Gobiernos Regionales (MINSA-GR), Seguro Social de Salud (EsSalud), Fuerzas Armadas y Policiales (FF.AA y PNP), y entidades prestadoras de salud, aseguradoras privadas, clínicas privadas y seguro universitario (CSP).

${ }^{*}$ Se denomina farmacia al establecimiento que es de propiedad de un profesional químico farmacéutico.

Todos los análisis consideraron la naturaleza compleja de la muestra.
} 
de que sus hijos recibieran muchos antibióticos, el 85,0\% estaba seguro de que existían consecuencias si se tomaban demasiados antibióticos y el 55,0\% mencionaron su preocupación acerca de la resistencia antibiótica ${ }^{(26)}$. Por otra parte, cabe la posibilidad que algunos padres no hayan admitido usar antibióticos sin receta médica en sus niños y esto subestimar la prevalencia real ${ }^{27}$. En el caso de la CASRM para el cónyuge, no se han encontrado estudios que evalúan los factores que se asocian, sin embargo, aplicando la misma lógica que para la CASRM para los hijos, esta baja prevalencia podría deberse a la preocupación de causar algún daño en la salud de los seres queridos y preferir la seguridad de que un profesional avale la prescripción para la dolencia que esté sufriendo en ese momento.

En el presente estudio, las variables que no se incluyeron en el análisis multivariable por no encontrarse significativamente asociadas fueron: grado de instrucción, actividad laboral e ingreso mensual. Estos factores se han visto previamente asociados en estudios como en el de Alhomoud et al (13), Jamhour et al (14) y Llanos-Zapata et al ${ }^{(28)}$ aunque debemos notar que estos estudios fueron realizados en poblaciones distintas y contextos diferentes. En nuestro estudio, a pesar de no encontrar asociación significativa con la variable grado de instrucción, se puede observar que existe un alto porcentaje de CASRM en todos los niveles de instrucción y que los que más CASRM son aquellos usuarios con superior universitaria completa y posgrado (aunque la diferencia no fue estadísticamente significativa). Se ha visto que tener un nivel de educación superior se asocia a automedicación, ya que creen tener conocimiento sobre los medicamentos que utilizan. Esto puede deberse a que tendrían mayor capacidad para interpretar los mensajes publicitarios de los diferentes medios de comunicación (21). Finalmente, respecto al ingreso económico, la CASRM fue similar entre los tres niveles de ingreso evaluados.

Como ya se ha descrito en la literatura, la falta de recursos económicos o falta de acceso a servicios de salud puede empujar a las personas a encontrar en la automedicación una forma de ahorro ${ }^{(13)}$. Además, por no perder su jornada de trabajo, recurren a esta práctica como una salida rápida, ya que acceder a una cita implicaría largas colas desde horas de la madrugada, largos días de espera para tener la consulta, así como exponerse a trato inadecuado por parte de los profesionales de la salud (22). Sin embargo, en el estudio de Llanos Zavala et al, se encontró que las personas en extrema pobreza, al no tener el dinero suficiente para adquirir los medicamentos, buscarían acudir a los establecimientos de salud, en donde les proporcionen medicamentos gratuitos de los programas nacionales o utilizando equivalentes en la medicina tradicional (28).

Nuestro estudio cuenta con ciertas limitaciones. Al ser un estudio de tipo transversal, la evaluación se ha centrado en la búsqueda de factores asociados, y no en el efecto causal de las exposiciones. Otra limitación respecto al diseño del estudio es que es un análisis secundario de datos y al no haber participado de la recolección de datos, surge cierta incertidumbre al momento de analizar si aquellos usuarios que compraron con receta médica lo hicieron realmente aconsejados por un profesional médico o mostraron una receta antigua o de algún familiar/amigo, ya que a esto también se le considera como automedicación. Asimismo, podría existir sesgo del entrevistador y deseabilidad social; sin embargo, los encuestadores fueron capacitados en la recolección de la información por los organizadores de la encuesta.

El estudio también tiene ciertas fortalezas. A nuestro conocimiento, es uno de los pocos estudios locales que evalúa la compra de antimicrobianos en un momento inmediatamente posterior a la misma, además de realizar la verificación verbal/visual de la receta. En cuanto al diseño de muestreo del estudio primario, se logró representatividad nacional, la cual constituye una de las mayores fortalezas del estudio. Es importante mencionar que la representatividad de nuestro estudio es a usuarios de farmacias y boticas que, exclusivamente, adquirieron al menos un antimicrobiano (no a población general, ni a la población de usuarios de farmacias y boticas en general), lo cual puede explicar la composición de la muestra.

En conclusión, se encontró que $53,4 \%$ de usuarios de boticas y farmacias compraron antimicrobianos sin receta médica. Encontramos una mayor prevalencia de CASRM en la sierra y en la selva; así como en los usuarios de farmacias. Mientras tanto, la prevalencia de CASRM fue menor en los usuarios asegurados al SIS y en quienes compraban el antimicrobiano para su cónyuge o hijo(a).

Por lo tanto, recomendamos reforzar el cumplimiento de la Ley General de Salud $N^{\circ} 26842$, con el fin que exista un control más estricto de todos los aspectos vinculados con el expendio de medicamentos (en especial, en la sierra y en la selva). Por otro lado, considerando que los usuarios del SIS tuvieron una menor prevalencia de CASRM, se debe continuar ampliando el acceso al aseguramiento hasta lograr la universalidad del mismo.

Contribución de autoria: CRAy PMT participaron en la concepción y diseño del estudio. Todos los autores participaron en el análisis e interpretación de los datos. CRA redactó la primera versión del manuscrito. RPE y PMT revisaron críticamente el mismo. Los tres autores aprobaron la versión final del artículo a publicar y asumen responsabilidad por su contenido.

Fuente de financiamento: Autofinanciada.

Conflictos de interés: Los autores declaran no tener conflictos de interés. 


\section{REFERENCIAS BIBLIOGRÁFICAS}

1. Azami-aghdash S, Mohseni M, Etemadi, M. Prevalence and Cause of SelfMedication in Iran : A Systematic Review and Meta-Analysis Article. Iran J Public Health. 2015;44(12):80-1593.

2. You J, Wong F, Chan F, Wong E, Yeoh E. Public perception on the role of community pharmacists in self-medication and self-care in Hong Kong. BMC Clin Pharmacol. 2011: 11-19.

3. Center for Disease Control and Prevention. Antibiotic resistance threats in the United States. Atlanta: CDC; 2013.

4. World Health Organization. Guidelines for the Regulatory Assessment of Medicinal Products for use in SelfMedication. Ginebra: Suiza; 2000.

5. Shoukat A, Ahmed J, Beekho G, Fayyaz $\mathrm{N}$, Zainulabdin Z, Jindani R. Practices of self-medication with antibiotics among nursing students of Institute of Nursing, Dow University of Health Sciences, Karachi, Pakistan. J Pak Med Assoc. 2016; 66(2):235-7.

6. Zhao Y, Ma S. Observations on the Prevalence, Characteristics, and effects of self-treatment. Front Public Health. 2016; 4:69.

7. Fajardo-Zapata A, Méndez-Casallas F, Hernández-Niño J, Molina L, Tarazona A, Nossa C, et al. La automedicación de antibióticos: un problema de salud pública. Sal Uni. 2013; 29(2):226-235.

8. Bilal M, Haseeb A, Khan M, Arshad M. Self-Medication with Antibiotics among People Dwelling in Rural Areas of Sindh. J Clin Diagn Resp. 2016; 10(5): 8-13.

9. Díaz-Caycedo N, Payán-Madriñán MA, Pérez-Acosta A. Aproximación psicológica al comportamiento de automedicación. Rev Cost Psico. 2014; 33(1): 17-29.

10. Sánchez J. Self-medication practices among a sample of Latino migrant workers in South Florida. Front Public Health. 2014; 2(108): 1-7.

11. Kumarasamy K, Toleman M, Walsh T, Bagaria J, Butt F, Balakrishnan R, et al. Emergence of a new antibiotic resistance mechanism in India, Pakistan, and the UK: a molecular, biological, and epidemiological study. Lancet Infect Dis. 2010; 10(9):597_ 602.

12. Al Rasheed A,Yagoub U, Alkhashan $\mathrm{H}$, Abdelhay O, Alawwad A, Aboud A, et al. Prevalence and Predictors of SelfMedication with Antibiotics in Al Wazarat Health Center, Riyadh City, KSA. BioMed Res Int. 2016; 2016:3916874.

13. Alhomoud F, Aljamea Z, Almahasnah $\mathrm{H}$, Alkhalifah K, Basalelah L, Almohoud FK. Self-medication and self-prescription with antibiotics in the Middle East - do they really happen? A systematic review of the prevalence, possible reasons, and outcomes. Int J Infect Dis. 2017;57: 3-12.

14. Monitoring and Evaluation of the Global Action Plan on Antimicrobial Resistance (AMR): Regional Expert Consultation on Monitoring and Evaluation of AMR Interventions. 2017; 26-27.

15. Gobierno del Perú. Ley General de Salud. Ley N²6842. Lima; Gobierno del Perú: 1997.

16. Hermoza-Moquillaza R, Loza-Munarriz C, Rodríguez-Hurtado D, Arellano Sacramento C, Hermoza-Moquillaza V. Automedicación en un distrito de Lima, Perú. Rev Med Hered. 2016;27:15-21.

17. Martínez L. Percepción de la automedicación con antibióticos en los usuarios externos en un hospital público en Lima [tesis]. Lima: Universidad Nacional Mayor de San Marcos. 2013.

18. Gutiérrez D, Romero C, Benites S. Dispensación de Antibióticos sin la presentación de la Receta Médica en Farmacias y Boticas, Centro Histórico de Trujillo, Perú. UCV-Scientia. 2015; $7(1): 44-51$

19. Instituto Nacional de Estadística e Informática. Encuesta Nacional de Satisfacción de Usuarios en Salud 2016. Informe Final. Dirección Nacional de Censos y Encuestas. INEI. Lima; 2016.

20. Gastelurrutia M, Larrañaga B, FernandezLlimos F. Impact of a program to reduce the dispensing of antibiotics without a prescription in Spain. Pharm Pract (Granada). 2013; 11(4):185-190.

21. Díaz M. Factores demográficos y socioeconómicos que se relacionan con la automedicación en la poblacion rural del distrito de Chota [Tesis]. Cajamarca: Universidad Nacional de Cajamarca. 2004.

22. Montoya G, Rubio T. Factores que influyen en la automedicación en el distrito de Villa Belén Zona Baja, Loreto [tesis]. Loreto: Universidad Nacional de la Amazonía Peruana. 2016

23. Ramos J. Caracterización de la práctica de automedicación en la población residente del distrito de Ate de la provincia de Lima [tesis]. Lima: Universidad Nacional Mayor de San Marcos. 2014.

24. Multicenter study on self-medication and self-prescription in six Latin American countries, Drug Utilization Research Group, Latin America. Clin Pharmacol Ther. 1997; 61(4):488-493.

25. Palmer DA, Bauchner H. Parents' and Physicians' Views on Antibiotics. Pediatrics. 1997; 99(6):E6.

26. Ecker L, Ruiz J, Vargas M, Del Valle LJ, Ochoa TJ. Prevalencia de compra sin receta y recomendación de antibióticos para niños menores de 5 años en farmacias privadas de zonas periurbanas en Lima, Perú. Rev Peru Med Exp Salud Publica. 2016;33(2):215-23.

27. Bauchner H, Pelton SI, Klein JO. Parents, Physicians, and Antibiotic Use. Pediatrics. 1999; 103(2): 395-401.

28. Llanos L, Contreras C, Velásquez J, Mayca J, Lecca L, Reyes R, Peinado J. Automedicación en cinco provincias de Cajamarca. Rev Med Hered. 2001; 12(4).

Correspondencia: Carolay Rojas-Adrianzén Dirección: Av. Costanera 2200 San Miguel Torre D - Dpto 506. Teléfono: 990990905 Correo electrónico: carolay.rojas.07@gmail.com 